Constantin Volintiru, Department of Mathematics, University of Bucharest, Bucharest, Romania. e-mail: cosv@mt.cs.unibuc.ro

\title{
A PROOF OF THE FUNDAMENTAL THEOREM OF CALCULUS USING HAUSDORFF MEASURES
}

\begin{abstract}
This note contains a proof of the Fundamental Theorem of Calculus for the Lebesgue-Bochner integral using Hausdorff measures (see 2.4). For the real case $(X=\mathbb{R})$, this proof uses only the basics from the Lebesgue integral theory (see 2.6).
\end{abstract}

\section{Preliminaries}

Throughout this paper, $\mu$ denotes the Lebesgue measure on $\mathbb{R}, X$ will be an arbitrary fixed real Banach space, and the measurability is understood relative to the Borel sets of $\mathbb{R}$ and $X$ respectively. We say that a given $f:[a, b] \mapsto$ $X$ is $\mu$-measurable if there exists the $\mu$-null Borel set $A \subseteq[a, b]$ such that $f([a, b] \backslash A)$ can be included in a separable subspace of $X$ and $f$ restricted to $[a, b] \backslash A$ is measurable. By an integral of a $\mu$-measurable $f:[a, b] \mapsto X$ we shall always mean the the Lebesgue integral or, to be more accurate on Banach spaces context, the Lebesgue-Bochner integral. Recall that there exists a (possibly infinite) integral $\int_{a}^{b} f(t) d t$ for every positive $\mu$-measurable function $f:[a, b] \mapsto \mathbb{R}_{+}$and, for a $\mu$-measurable $f:[a, b] \mapsto X$, one says that $f$ is $\mu$-integrable if and only if $\int_{a}^{b}\|f(t)\| d t<+\infty$, and in this case the integral $\int_{a}^{b} f d t$ exists. Obviously, $[a, b]$ could be replaced with an arbitrary interval of reals.

It has to be pointed out that apart from basic definitions from integration theory, we shall use in the sequel almost nothing but the following easy consequence of the Dominated Convergence Theorem.

\footnotetext{
Key Words: Fundamental Theorem of Calculus, Hausdorff measures

Mathematical Reviews subject classification: 26A42, 26A46, 28A78

Received by the editors April 2, 1999
} 
Proposition 1.1. If $f:[a, b] \mapsto X$ and $f_{n}:[a, b] \mapsto X(n \geq 1)$ is a sequence of integrable functions such that $f_{n}(t) \mapsto f(t)$ a.e. , then $f$ is integrable and

$$
\int_{a}^{b} f_{n}(t) d t \longmapsto \int_{a}^{b} f(t) d t
$$

provided that $\left(f_{n}\right)_{n}$ is uniformly integrable.

Note : Recall that the uniform integrability of $\left(f_{n}\right)_{n}$ means

$$
\lim _{k \mapsto+\infty} \int_{\left\|f_{n}\right\| \geq k}\left\|f_{n}(t)\right\| d t=0 \quad \text { uniformly for } \quad n \geq 1
$$

and in this case the fact that $[a, b]$ is a bounded interval is essential because the proof of the above proposition needs finite measure.

All the above are standard facts in this field and can be found in almost every measure theory books - see for example [2] and [7].

A definition of the Hausdorff measure $h_{\alpha}$ would go as follows: if $(T, d)$ is any metric space, $A \subseteq T$ and $\delta>0$, let $\Lambda(A, \delta)$ be the set of all arbitrary collections $(C)_{i}$ of subsets of $T$, such that $A \subseteq \bigcup_{i} C_{i}$ and $\operatorname{diam}\left(C_{i}\right) \leq \delta$ for every $i$. Now, for every $\alpha>0$ define

$$
h_{\alpha}^{\delta}(A) \stackrel{\text { def }}{=} \inf \left\{\sum\left(\operatorname{diam} C_{i}\right)^{\alpha} \quad \mid \quad\left(C_{i}\right)_{i} \in \Lambda(A, \delta)\right\} .
$$

Then there exists $\lim _{\delta \mapsto 0} h_{\alpha}^{\delta}(A)=\sup _{\delta>0} h_{\alpha}^{\delta}(A)$ and $h_{\alpha}(A) \stackrel{\text { def }}{=} \lim _{\delta \mapsto 0} h_{\alpha}^{\delta}(A)$ gives an outer measure on $\mathcal{P}(T)$ which is countable additive on the $\sigma$-field of all Borel subsets of $T$.

If $T=\mathbb{R}^{n}$, the Hausdorff measure $h_{n}$, restricted to the $\sigma$-field of the Borel subsets of $\mathbb{R}^{n}$, is identical to the Lebesgue measure on $\mathbb{R}^{n}$ up to a constant multiple. In particular, $h_{1}(C)=\mu(C)$ for every Borel set $C \subseteq \mathbb{R}$. With this remark, the following proposition is the translation of a well known inequality:

Proposition 1.2. If $C \subseteq \mathbb{R}$ is a Borel set and $F: C \longmapsto X$ is a Lipschitz map (i.e $\|f(x)-f(y)\| \leq a|x-y|$ whenever $x, y \in C$ ), then

$$
h_{1}(F(C)) \leq a \mu(C) .
$$

Apart from the above proposition, the next result is the only one needed in this paper from the Hausdorff measure theory. See for example [4] or [3].

Proposition 1.3. If $(T, d)$ is a metric space and $C \subseteq T$ is connected, then

$$
\operatorname{diam}(C) \leq h_{1}(C) .
$$


We conclude this section with some notations.

Throughout this paper we fix, for an arbitrary interval $[a, b]$ with $a<b$, a sequence of divisions

$$
\Delta_{n}=\left\{a=t_{0}^{(n)}<t_{1}^{(n)}<\cdots<t_{k_{n}-1}^{(n)}<t_{k_{n}}^{(n)}=b\right\} \quad \text { for } \quad n \geq 1
$$

such that $\Delta_{n} \leq \Delta_{n+1}$ in the sense that every $t_{i}^{n}$ from $\Delta_{n}$ can be found as some $t_{j}^{(n+1)}$ in $\Delta_{n+1}$, and

$$
\delta_{n} \stackrel{\text { def }}{=} \underset{i=0}{\max _{n}-1}\left(t_{i+1}^{(n)}-t_{i}^{(n)}\right) \longmapsto 0
$$

For every $g:[a, b] \mapsto X$ and $n \geq 1$, let

$$
\Delta_{n} g=\sum_{i=0}^{k_{n}-1} \frac{g\left(t_{i+1}^{(n)}\right)-g\left(t_{n}^{(n)}\right)}{t_{i+1}^{(n)}-t_{i}^{(n)}} \mathbf{1}_{\left[t_{i}^{(n)}, t_{i+1}^{(n)}\right)}
$$

where, for any $C \subseteq[a, b], \mathbf{1}_{C}(t)=1$ if $t \in C$ and $\mathbf{1}_{C}(t)=0$ if $t \notin C$.

Remark 1.4. For the given $F:[a, b] \longmapsto X$ and $f:[a, b] \longmapsto X$, if the derivative $F^{\prime}(t)$ exists a.e. and $F^{\prime}(t)=f(t)$ a.e. , then

$$
\Delta_{n} F(t) \longmapsto f(t) \quad \text { a.e. }
$$

Indeed, if $F^{\prime}(t)=f(t)$ and $t \notin \bigcup_{n} \Delta_{n}$, i.e. almost everywhere, then there exists an uniquely defined sequence of open intervals $\left(a_{n}, b_{n}\right)_{n \geq 1}$ such that $a_{n}, b_{n}$ are consecutive points in $\Delta_{n}$ and $t \in\left(a_{n} . b_{n}\right)$ for every $n$. Since $b_{n}-a_{n} \longmapsto 0$ by 3 ,

$$
\Delta_{n} F(t)=\frac{F\left(b_{n}\right)-F\left(a_{n}\right)}{b_{n}-a_{n}} \longmapsto f^{\prime}(t) .
$$

The final aim of this proof for FTC is to show that under some conditions, $\left(\Delta_{n} F\right)_{n}$ is uniformly integrable.

\section{Main result}

We fix $f:[a, b] \mapsto X$, a continuous function $F:[a, b] \longmapsto X$, and we assume that there exists a Borel set $B \subset[a, b]$ such that:

the derivative $F^{\prime}(t)$ exists and $F^{\prime}(t)=f(t)$ for every $t \in[a, b] \backslash B$. 
In the end, $\mu(B)=0$ will be an additional hypothesis, but this is of no consequence for the moment.

Since $f(t)=F^{\prime}(t)$ for $t \in[a, b] \backslash B, f$ restricted to $[a, b] \backslash B$ is measurable, therefore $\|f\|$ restricted to $[a, b] \backslash B$ is measurable, any set of the form

$$
C=\{t \in[a, b] \backslash B \mid\|f(t)\| \leq \rho\}
$$

is a Borel set, the (possibly infinite) integral $\int_{C}\|f(t)\| d t$ exists, and so on.

Lemma 2.1. For any fixed $\rho>0$ there exists a sequence of mutually disjoint Borel sets $\left(C_{k}\right)_{k \geq 1}$, such that

$$
[a, b] \backslash B=\bigcup_{k \geq 1} C_{k}
$$

and for every $k \geq 1$,

$$
\|F(x)-F(y)-(x-y) f(y)\| \leq \rho|x-y| \quad \text { whenever } \quad x, y \in C_{k} .
$$

Proof. For every fixed rational $t \in \mathbb{Q}$ denote by $A_{t}$, the set of all those $y \in[a, b] \backslash B$ such that

$$
y+t \in[a, b] \text { and }\|F(y+t)-F(y)-t f(y)\| \leq \rho|t| \quad .
$$

Since $f$ restricted to $[a, b] \backslash B$ is measurable and $F$ is continuous, the map

$$
y \mapsto F(y+t)-F(y)-t f(y)
$$

is a measurable function on the set

$$
([a, b] \backslash B) \cap\{y \mid y+t \in[a, b]\} .
$$

It follows that each $A_{t}$ is a Borel set and, with the sequence $\left(\delta_{n}\right)_{n}$ given by 3 ,

$$
B_{n} \stackrel{\text { def }}{=} \bigcap_{t \in \mathbb{Q},|t| \leq \delta_{n}} A_{t}
$$

is a Borel set, as an intersection of a countable family of Borel sets. Since $F$ is continuous and $x=y+(x-y)$,

$y \in B_{n}$ if and only if

$$
\|F(x)-F(y)-(x-y) f(y)\| \leq \rho|x-y|
$$

for every $x \in[a, b]$ such that $|x-y| \leq \delta_{n}$, 
and from $F^{\prime}(y)=f(y)$ for $y \in[a, b] \backslash B$, it follows

$$
[a, b] \backslash B=\bigcup_{n \geq 1} B_{n} .
$$

Let $E_{1}=B_{1}, E_{2}=B_{2} \backslash E_{1}, \ldots, E_{n}=B_{n} \backslash\left(E_{1} \cup E_{2} \cup \cdots \cup E_{n-1}\right), \ldots$

The Borel sets $E_{n}(n \geq 1)$ are mutually disjoint,

$$
E_{n} \subseteq B_{n} \quad(n \geq 1) \quad \text { and } \quad \bigcup_{n \geq 1} E_{n}=\bigcup_{n \geq 1} B_{n}=[a, b] \backslash B
$$

Finally, for every $n \geq 1$, let $\Delta_{n}$ be the division given by 2 , and define

$$
\begin{gathered}
E_{n}^{(i)}=E_{n} \cap\left[t_{i}^{(n)}, t_{i+1}^{(n)}\right) \quad \text { if } \quad 0 \leq i<k_{n}-1, \\
\text { and } E_{n}^{\left(k_{n}-1\right)}=E_{n} \cap\left[t_{k_{n}-1}^{(n)}, t_{k_{n}}^{(n)}\right] .
\end{gathered}
$$

The sequence $\left(C_{k}\right)_{k \geq 1}$ is the result of an arbitrary enumeration of the sets $E_{n}^{(i)}$. Indeed, if $C_{k}=E_{n}^{(\bar{i})}$ for some $n \geq 1$ and $0 \leq i \leq k_{n}-1$, then $|x-y| \leq \delta_{n}$ for every $x, y \in C_{k}$,

$$
C_{k}=E_{n}^{(i)} \subseteq E_{n} \stackrel{\text { from } 7}{\subseteq} B_{n}
$$

and 5 is an easy consequence of 6 .

Remark 2.2. Within the hypothesis of the preceding lemma, since 5 is symmetric for any fixed $C_{k}$ and $x, y \in C_{k}$, we have $\|F(y)-F(x)-f(x)(y-x)\| \leq$ $\rho|y-x|$, therefore

$$
\begin{gathered}
|x-y|\|f(x)-f(y)\|=\|-f(y)(x-y)-f(x)(y-x)\| \leq \\
\leq\|F(x)-F(y)-f(y)(x-y)\|+\|F(y)-F(x)-f(x)(y-x)\| \leq \\
\leq 2 \rho|x-y|,
\end{gathered}
$$

hence $\|f(x)-f(y)\| \leq 2 \rho$ for every $x, y \in C_{k}$ so, if we define

$$
a_{k}=\inf \left\{\|f(t)\| \mid t \in C_{k}\right\} \text {, }
$$

then $\quad 0 \leq a_{k} \leq\|f(x)\| \leq a_{k}+2 \rho \quad$ for every $\quad x \in C_{k}$.

Lemma 2.3. If $\left(C_{k}\right)_{k \geq 1}$ is the sequence given by lemma 2.1 for $\rho>0$, then for every $k \geq 1$,

$$
h_{1}\left(F\left(C_{k}\right)\right) \leq 3 \rho \mu\left(C_{k}\right)+\int_{C_{k}}\|f(t)\| d t .
$$


Proof. Let $\left(a_{k}\right)_{k \geq 1}$ be given by 2.2 , and fix some $C_{k}$. If $x, y \in C_{k}$,

$$
\begin{gathered}
\|F(x)-F(y)\| \stackrel{\text { from } 5}{\leq}(\rho+\|f(y)\|)|x-y| \stackrel{\text { by } 8}{\leq}\left(3 \rho+a_{k}\right)|x-y|, \\
\text { hence } h_{1}\left(F\left(C_{k}\right)\right) \stackrel{\text { by }}{\leq} \leq\left(3 \rho+a_{k}\right) \mu\left(C_{k}\right) .
\end{gathered}
$$

Since $a_{k} \leq\|f(t)\|$ for every $t \in C_{k}$, with $\mathbf{1}_{C_{k}}(t)=1$ for $t \in C_{k}$ and $\mathbf{1}_{C_{k}}(t)=0$ if $t \notin C_{k}$, we have

$$
\begin{gathered}
\left(3 \rho+a_{k}\right) \mathbf{1}_{C_{k}} \leq 3 \rho \mathbf{1}_{C_{k}}+\|f\| \mathbf{1}_{C_{k}} \quad, \quad \text { therefore } \\
\left(3 \rho+a_{k}\right) \mu\left(C_{k}\right)=\int_{a}^{b}\left(3 \rho+a_{k}\right) \mathbf{1}_{C_{k}}(t) d t \leq \\
\leq 3 \rho \int_{a}^{b} \mathbf{1}_{C_{k}}(t) d t+\int_{a}^{b}\|f(t)\| \mathbf{1}_{C_{k}}(t) d t=3 \rho \mu\left(C_{k}\right)+\int_{C_{k}}\|f(t)\| d t .
\end{gathered}
$$

Finally, $\quad h_{1}\left(F\left(C_{k}\right)\right) \stackrel{\text { by } 9}{\leq}\left(3 \rho+a_{k}\right) \mu\left(C_{k}\right) \leq 3 \rho \mu\left(C_{k}\right)+\int_{C_{k}}\|f(t)\| d t$.

Proposition 2.4. (Fundamental Theorem of Calculus) Suppose that for the given

$$
f:[a, b] \longmapsto X
$$

there exists $F:[a, b] \mapsto X$, which is continuous, the derivative $F^{\prime}(t)$ exists and $F^{\prime}(t)=f(t)$ outside a $\mu$-null Borel set $B \subseteq[a, b]$ such that

$$
h_{1}(F(B))=0 \quad .
$$

Then $f$ is $\mu$-measurable and if we assume the integrability of $f$,

$$
F(b)-F(a)=\int_{a}^{b} f(t) d t
$$

Proof. Let $F:[a, b] \mapsto X$ and $B \subseteq[a, b]$ as above. Recall that the integrability of the ( $\mu$-measurable) $f:[a, b] \mapsto X$ is equivalent to

$$
\int_{a}^{b}\|f(t)\| d t<+\infty
$$


Let $\left(C_{k}\right)_{k>1}$ be the sequence given by lemma 2.1 for an arbitrary $\rho>0$. Since $\mu(B)=0$ and the sets $C_{k}$ are mutually disjoint, $b-a=\sum_{k \geq 1} \mu\left(C_{k}\right)$ and

$$
\begin{gathered}
h_{1}(F([a, b]))=h_{1}\left(F(B) \cup\left(\bigcup_{k \geq 1} F\left(C_{k}\right)\right)\right) \stackrel{h_{1}(F(B))=0 \text { by } 10}{\leq} \\
\leq \sum_{k \geq 1} h_{1}\left(F\left(C_{k}\right)\right) \stackrel{b y}{\leq} \sum_{k \geq 1}^{2.3}\left[3 \rho \mu\left(C_{k}\right)+\int_{C_{k}}\|f(t)\| d t\right] \leq \\
\leq 3 \rho(b-a)+\int_{a}^{b}\|f(t)\| d t<+\infty
\end{gathered}
$$

for every $\rho>0$, therefore

$$
h_{1}(F([a, b])) \leq \int_{a}^{b}\|f(t)\| d t \quad .
$$

By the continuity of $F$, the set $F([x, y])$ is connected in $X$, hence

$$
\|F(b)-F(a)\| \leq \operatorname{diam}(F([a, b])) \stackrel{b y}{\leq} \leq h_{1}(F([a, b]))
$$

which, taking into account 13, gives:

$$
\|F(b)-F(a)\| \leq \int_{a}^{b}\|f(t)\| d t .
$$

Since the same argument as above can be applied for every interval $[x, y] \subseteq$ $[a, b]$ instead of $[a, b]$, if we define $\varphi:[a, b] \longmapsto \mathbb{R}_{+}$, by

$$
\varphi(x)=\int_{a}^{x}\|f(t)\| d t \quad \text { for } \quad a \leq x \leq b
$$

from 14 we have

$$
\|F(y)-F(x)\| \leq \varphi(y)-\varphi(x) \quad \text { for } \quad a \leq x<y \leq b
$$

With the notations from the last part of section 1 , it follows that

$$
\left\|\Delta_{n} F(t)\right\| \leq \Delta_{n} \varphi(t) \quad \text { for every } \quad t \in[a, b]
$$

As a consequence of the definition $15,\left(\Delta_{n} \varphi\right)_{n}$ is uniformly integrable, so $\left(\Delta_{n} F\right)_{n}$ is uniformly integrable by 16 and $\Delta_{n} F(t) \longmapsto f(t)$ a.e. by 1.4 . It 
remains to observe that

$$
\begin{gathered}
\int_{a}^{b} f(t) d t \stackrel{b y}{=} \lim _{n} \int_{a}^{b} \Delta_{n} F(t) d t= \\
=\lim _{n}\left[\sum_{i=0}^{k_{n}-1}\left(F\left(t_{i+1}^{(n)}\right)-F\left(t_{i}^{(n)}\right)\right]=\lim _{n}[F(b)-F(a)]=F(b)-F(a) .\right.
\end{gathered}
$$

The choice of a bounded interval $[a, b]$ is due only to the fact that this includes the hard part of the above proofs. Since an arbitrary interval of reals is the union of a sequence of bounded intervals, the following proposition is the result of 2.4 via some standard arguments:

Proposition 2.5. Let $I \subseteq \mathbb{R}$ be an arbitrary interval, with $u=\sup I$ and $v=\inf I$. Suppose that for the given $f: I \longmapsto X$, there exists $F: I \mapsto X$, which is continuous, the derivative $F^{\prime}(t)$ exists and $F^{\prime}(t)=f(t)$ outside a $\mu$-null Borel set $B \subseteq I$ such that $h_{1}(F(B))=0$.

Then $f$ is $\mu$-measurable and if $f$ is integrable, then $F(v-0), F(u+0) \in X$ exist and

$$
F(v-0)-F(u+0)=\int_{u}^{v} f(t) d t
$$

Remark 2.6. The previous propositions include the situation $X=\mathbb{R}$, in which case the condition $h_{1}(F(B))=0$ is equivalent with $\mu(F(B))=0$, where $\mu$ stands for the Lebesgue outer measure on $\mathbb{R}$.

In this case an explicit use of the Hausdorff measure theory is not necessary in the above proof - the inequality from 1.2 becomes $\mu((F(C)) \leq a \mu(C)$ and, instead of 1.3 , it suffices to observe that any connected subset $C \subset \mathbb{R}$ is always an interval, so $\operatorname{diam}(C)=\mu(C)$. With this observation, the entire real case proof for FTC presented in this paper becomes fully accessible in an introductory course of measure theory.

Remark 2.7. In the case of a positive $f:[a, b] \longmapsto \mathbb{R}_{+}$the function $F$ from 2.4 is monotone and the integrability of $f$ is the result of the inequality $\int_{a}^{b} f(t) d t \leq$ $F(b)-F(a)<+\infty$.

Consequently, in the proposition 2.5 , if $f: I \longmapsto \mathbb{R}_{+}$, the integrability condition is not necessary and 17 holds, with $F(v-0)-F(u+0)=+\infty$ being a possibility.

It should be pointed out that this particular case can be viewed as an independent result coming from another ideas. If follows from lema 2.1, applied to $F$ for a fixed $\rho>0$, that $F$ restricted to every $C_{k}$ is a Lipschitz map, therefore $\mu(F(C))=0$ for every $\mu$-null set $C \subset[a, b]$, as a consequence of the condition 
$\mu(F(B))=0$, i.e. $F$ verifies the Luzin condition $(N)$, hence $F$ is absolutely continuous by Banach-Zarecki Theorem ( see for example [1] ). Finally, 11 holds as a direct consequence of the Lebesgue-Radon-Nikodym Theorem.

\section{Comments}

The author of this note happens to be fond of Hausdorff measures, but has no serious informations on the FTC research area, and this proof has been found almost by accident. Although for the real case $(X=\mathbb{R}), 2.4$ cannot be explicitly found in Saks's book (see [6]), it is there, somewhat between the lines, ultimately as a consequence of the Theorem 7.7, page 285. Perhaps because of the fact that the arguments given in [6] are full of technicalities, apparently 2.4 became almost absent in the subsequent general presentations of the Lebesgue integral. The case $B=\emptyset$, i.e. $F^{\prime}(t)$ exists everywhere in $[a, b]$, is much better known - see [5] for example.

The author wishes to thank to professor Brian S. Thompson for the observation ( made in the real-analysis mailing list ) that 2.4 can be somehow found in Saks's book, and to the referee for many helpful remarks made on the previous version of this paper.

\section{References}

[1] A. M. Bruckner, B. S. Thompson, J. Bruckner, Real Analysis, Prentice Hall, 1996.

[2] J. Diestel, J.J.Uhl Jr, Vector Measures, Math. Surveys 15, Amer. Math. Soc. 1977.

[3] G. A. Edgar, Measure, Topology, and Fractal Geometry, Springer-Verlag, 1990.

[4] G. Federer, Geometric Measure Theory, Springer-Verlag, 1968.

[5] W. Rudin, Real and Complex Analysis, third edition. McGraw-Hill, 1987.

[6] S. Saks, Theory of the Integral, Hafner Publishing Co., 1937.

[7] E. Schechter, Handbook of Analysis and its Foundations, Academic Press, 1996. 
